\section{INSTALLATIONS HYDRO-ELECTRIQUES}

\section{ROULS PELTON DE L'ALUHINIUI CORPORATION}

Ces roues ont été construites par la maison Ganz et Cie, de Budapest, pour la station d'énergie électrique de Conway, de l'Aluminium Corporation. Cetle station est équipée avec 5 turbines Pelton, de $\overline{\mathrm{I}} 500 \mathrm{HP}$ chacune, qui actionnent 5 paires de dynamos pour la production du courant continu.

Chaque turbine sc compose de deux roues de $\mathrm{I}^{\mathrm{m}} / \mathrm{j} 0 \mathrm{o}$ de dia-
Le collecteur est placé en dehors du bâtiment, dans une fosse couverte de $\mathrm{r}^{\mathrm{m}} 250$ de profondeur. L'eau de sortie s'écoule en une nappe de $\mathrm{x}$. de hauteur par une galerie souterraine de ${ }^{\mathrm{m}} 990$ de largeur, disposée sous les moteurs.

Les détails de construction des turbines sont donnés par les figures ci-jointes. Chaque turbine est contenue dans une enveloppe en fonte en plusieurs parties ; deux d'entre elles forment le bâti de l'appareil, et se trouvent boulonnées sur les pierres de fondation. Les autres parties forment le couvercle des roues, elles sont munies d'ceillets de suspension, qui permettent de les soulever.

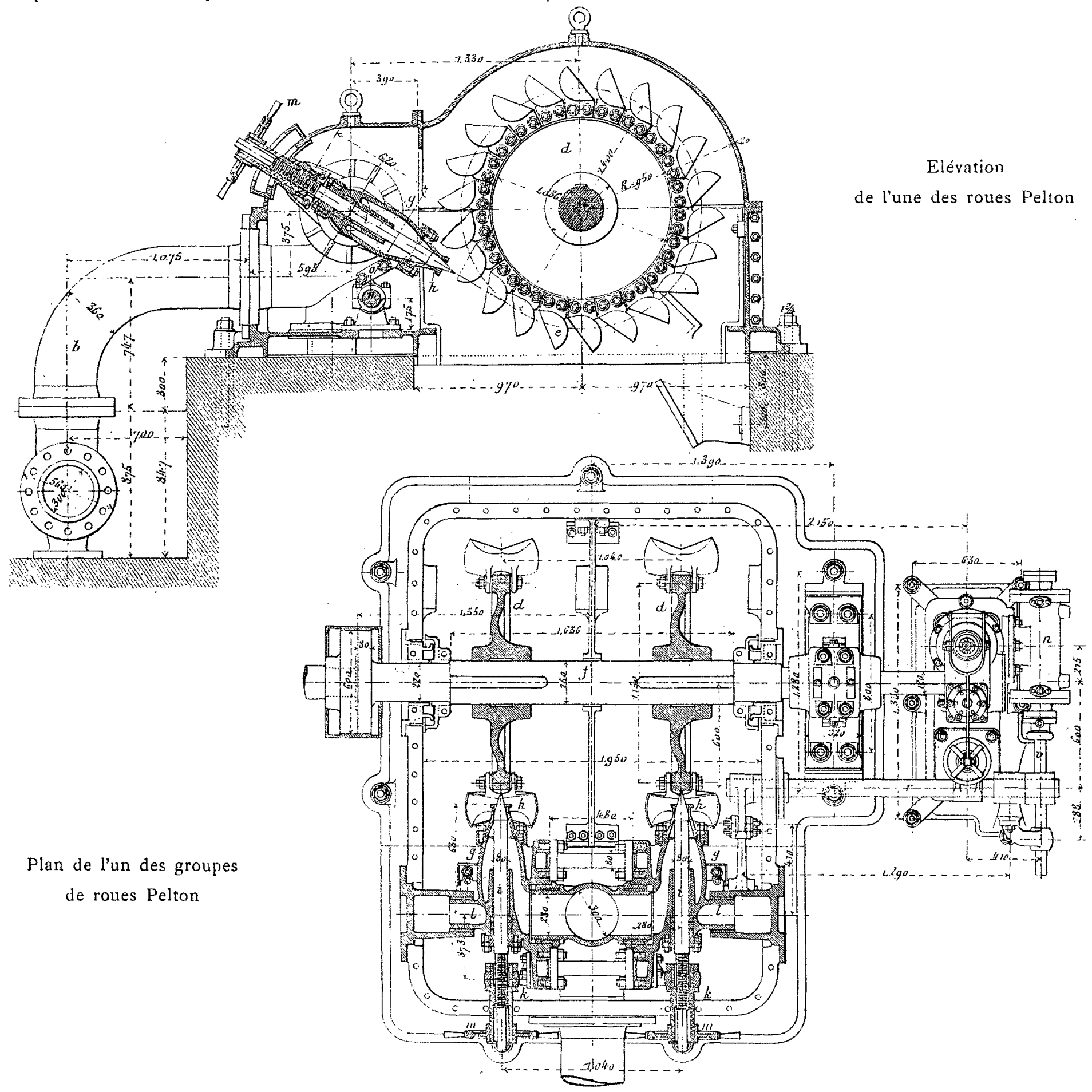

mètre ; elle fait 450 tours par minule, sous $290 \mathrm{~m}$. de chute, et absorbe 520 litres par seconde.

L'ean arrive par une conduite principale, de $\mathrm{r}^{\mathrm{m}}$ or 6 de diamètre intérieur, sur laquelle sont branchées des conduites dérivées $b$, de $300 \mathrm{~m}$. de diamètre intérieur, amenant l'eau jusqu'aux roues, et pourvues d'une vanne pour intercepter la communication, afin d'isoler la turbine correspondante.
Chaque moleur comporte deux roues, comme on l'a déjà dit ; ces deux roues, calées sur un même arbre horizontal $f$, se trouvent à $\mathrm{I}^{\mathrm{m}} \mathrm{o}$ /o d'axe en axe l'une de l'autre ; l'arbre a $250 \mathrm{~mm}$. de diamètre au calage des roues, et $220 \mathrm{~mm}$. à la portée dans les paliers ; d'un côté, i] est muni d'un manchon d'accouplement, de 6 oo $\mathrm{mm}$. de diamètre et de $80 \mathrm{~mm}$. d'épaisseur, par lequel il est relié au groupe électrogène. 
L'eau arrive sur chaque pàire de roues par deux tuyères accouplées ; chaque tuyère comprend une cage, ou corps en fonte $g$, terminé par un ajutage en bronze $h$, dans lequel sajuste un pointeau $i$, manoeurré par un volant à main $m$. Les deux tuyères $g$ pivotent par des tourillons $l$ sur des supports fixés au bâti ; elles sont solidement entretoisées entro elles de manière à former un tout solidaire emboîtant exactement la pièce de raccord en T par laquelle arrive l'eau ; un joint étanche est interposé entre chaque tuyère et cette pièce de raccord.

Le réglage du débit des tuyères s'effectue à la main par la manœurre du pointeau $i$, et leur changement de position par rapport aux roues a lieu automatiquement par le régulateur, disposé à droite de chaque moteur, et comprenant un cylindre $u$, dans lequel arrive de l'eau sous pression empruntéc directement à la conduite principale. La tige $v$ du piston de ce cylindre agit par un coulisseau sur un levier calé sur l'arbre $r$, lequel, par l'intermédiaire d'autres leviers, fail biellettes $o$.

M. $P$.

\section{USINE A BaSSE CHUTE DE LA TIPPECANOE RIVER}

La Tippecanoe Electric \& Power $C^{\circ}$ a aménagé à Monticello, dans le White County (Indiana), une chute de $2^{\mathrm{m}} 44$ de la rivière Tippecanoe, dont le débit est assez régulier et varie au plus de $\mathrm{Ir} \mathrm{m} \mathrm{m}^{3}$ en basse eaux à $45 \mathrm{~m}^{3}$ en temps de crues. L'installation comprend un l)arrage à caisson on bois rempli de cailloux, qui se prolonge d'un còté par l'usine hydro-électrique, et de l'autre par un ensemble de 3 vannes d'évacuation du type TainLer, de $3^{\mathrm{m}} 66$ de hauteur $\left(^{*}\right)$.

Le barrage est établi aux lieu et place d'un ancien barrage de moulin, dont il utilise les fondations. Le profit de ce vieux barrage est représenté sur la figure I par une ligne pointillée (old dam). Il est constitué par un caisson, formé de madriers de $o^{\mathrm{m}} 305$ de côté, chevillís ensemble, dont les vides sont remplis de cailloux (boulder fill). Les parements sont constitués par des planches de ${ }^{\mathrm{m}} 305$ de largeur et $76 \mathrm{~mm}$. d'épaisseur, cloućes sur les

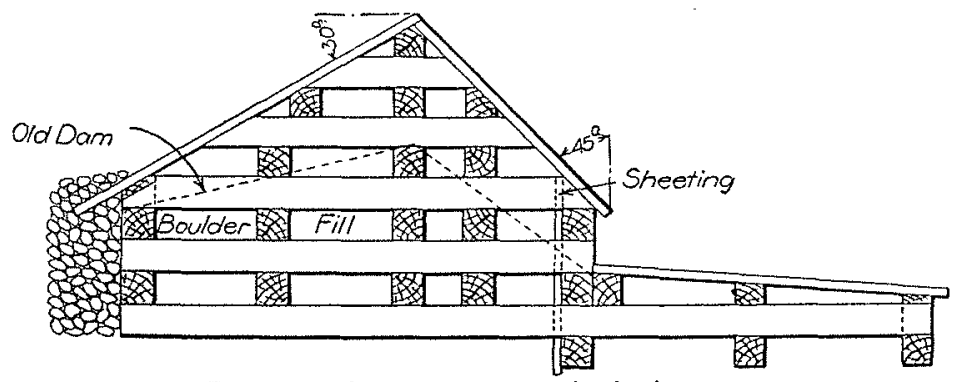

Fig. 1. - Coupe transversale du barrage,

madriers extérieurs. Le parement amont est incliné de $30^{\circ}$ sur l'horizontale, et celui d'aval de $45^{\circ}$. Des palplanches (sheeting) ont été descendues à travers le gravier jusqu'au rocher de fondation.

Les vannes mobiles sont au nombre de 3 , et ont été établies à I'une des extrémités du barrage, du côté opposé à l'usine, parce que la rivière forme un coude à cet endroit.

(*) D'après l'Engineering Record.
On a placé les vannes sur le côté concave, où les graviers et les glaces sont naturellement entrâinées par le courant qui est le plus fort de cè côté. Ceś vannes, qui sont représentées sur la figure 2, sont actionnées chacune par un moteur électrique de 2 IIP, qui est commandé de l'usine mème $\left(^{*}\right)$ par un càble étanche qui tuaverse le barrage dans toute sa longueur. Lo moteur tourne à I. I fo tours par mi-

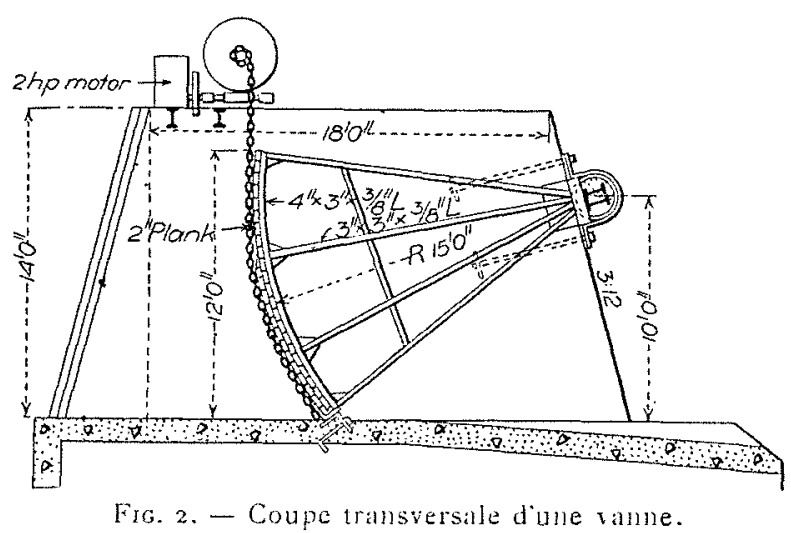

nulc, ct un syslème d'engrenages droits et à vis sans fin ramène à 2 le nombre de tours de l'arbre de commande des vannes.

Larbre de rotation de ces vannes mérite une mention spéciale. Il est constitué par deux fers à double $\mathrm{T}$, de 38 r mm. de hauteur, qui, à chaque extrémité, sont emmanchés lous deux dans un tube de chaudière, dont la paroie

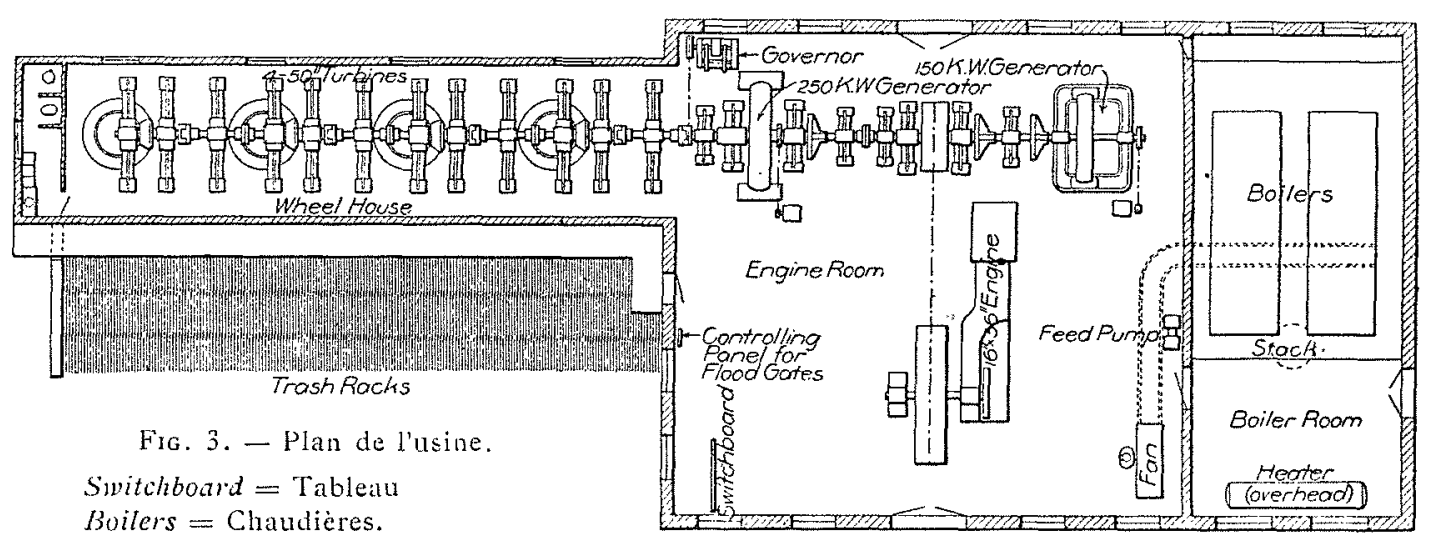

cxtérieure forme portée. L'espace libre entre le lube et les fers à $T$ est rempli de mortier de ciment.

L'usine comprend 4 lurbines verticales, dont les roues mobiles ont $\mathbf{I}^{m} 25$ de diamètre, qui actionnent par engrenages coniques un mème arbre horizontal, tournant à i 55 tours par minule, sur lequel sont montés deux alternateurs triphasés, de $\mathrm{r} 50$ et $250 \mathrm{kw}$, sous 2 ooo volts, 60 périodes par seconde. Une machine à vapeur de réserve complète l'inslallation. On peut à volonté débrayer ou embrayer l'une quelconque des lurbines, ainsi que la machine à vapeur.

L'usine, ainsi que les piles des vannes mobiles, sont en béton armé.

Un seul homme suffit pour assurer la marche de l'usine quand la machine à vapeur ne fonctionne pas. Celle-ci n'est mise en marche qu'en temps de crues, lorsque la hauteur de chnte diminue par trop, on bien en cas de réparation à l'une des turbines. Dans ce cas, on débraye le régulateur des turbines (governor), et le réglage de la vitesse est effectué par le régulateur do la machine à vapeur.

H. $B$.

(*) Le pannean de commande de ces moteurs est représenté sur la fig. 3 (Controlling panel for flood gates). 